\title{
Charge transport and morphology of pentacene films confined in nano-patterned region
}

\begin{abstract}
Tae-Sik Kim ${ }^{1}$, Se Hyun Kim², Mi Jang ${ }^{3}$, Hoichang Yang ${ }^{3}$ and Tae-Woo Lee ${ }^{1}$
The transport and morphology of macroscopic organic transistors have been thoroughly explained. However, the relationship between charge transport and the morphology of organic semiconductors in nano-confined regions is not well understood, which is required to design high-performance nano-electronics. Therefore, in this study, the electrical performance of pentacene thin-film transistors (TFTs) fabricated on nano-scale confined geometries was compared with that of other TFT systems fabricated on micro-scale confined or flat dielectrics. The results showed that the photoresist (PR) reliefs patterned onto silicon dioxide $\left(\mathrm{SiO}_{2}\right)$ dielectrics could control the growth mode of pentacene in the confined regions. As the line spacing between the PR patterns decreased to below the average size of pentacene normally grown on $\mathrm{SiO}_{2}$ dielectrics $(\sim 1.7 \mu \mathrm{m})$, field-effect mobility $\left(\mu_{\mathrm{FET}}\right)$ of pentacene TFTs improved considerably. Specifically, the $\mu_{\mathrm{FET}}$ values for the $250 \mathrm{~nm}$ patterned dielectric system were as high as $0.33 \mathrm{~cm}^{2} \mathrm{~V}^{-1} \mathrm{~s}^{-1}$, which was greater than those of the wider-patterned PR or flat dielectric systems $\left(\leqslant 0.15 \mathrm{~cm}^{2} \mathrm{~V}^{-1} \mathrm{~s}^{-1}\right)$. These findings were related to the enhancement of charge-carrier transport owing to the anisotropic $\pi$-conjugated crystals nucleated from the nano-confined edges, providing important information that can be used in the design of high-performance nano-scale organic electronics.
\end{abstract}

NPG Asia Materials (2014) 6, e91; doi:10.1038/am.2014.3; published online 14 March 2014

Keywords: crystallinity; growth mode; nano-confined region; OTFTs; pentacene

\section{INTRODUCTION}

The possibility of using organic semiconductors for electronic applications has been of great scientific and technological interest for several decades. The need for flexible electronics such as flexible displays, radio frequency identification tags and smart cards has been increasing because of the practical use of organic semiconductors in optoelectronic applications. The charge-carrier mobility of organic thin-film transistors (OTFTs) that use small molecules has improved considerably in response to optimization of deposition conditions, such as the deposition rate, ${ }^{1,2}$ substrate temperature, ${ }^{3}$ roughness ${ }^{4}$ and surface treatment. ${ }^{5,6}$ Organic electronic devices have also been reduced in size to the nano-scale and investigated for nextgeneration nano-electronic applications..$^{7-11}$ Self-assembled nanostructures are of fundamental interest for understanding the structure-dependent properties of $\pi$-conjugated organic semiconductors. ${ }^{12,13}$ Molecular packing in the highly organized onedimensional (1D) nano-structure of organic semiconductors is critical for determining charge transport mechanisms ${ }^{14}$ and the rational design of high-performance nano- and micro- electronic devices. However, to clarify the effects of active layer morphology on charge transport in organic semiconductor nano-devices, related studies must be conducted at the nano-scale. Here, we investigated how the morphology of pentacene grown in nano- and microconfined line patterns affects charge transport in OTFT, comparing with normal pentacene morphology with a terrace-like feature and an average grain size of $\sim 1.7 \mu \mathrm{m}$. To systematically investigate how the nano-confinement affects pentacene film growth and charge transport along electrodes, micro- and nano-confined photoresist (PR) patterns were fabricated by conventional photolithography and phase-shift soft lithography using a transparent poly(dimethylsiloxane) (PDMS) mold. Height of all the PR patterns were designed to be above $250 \mathrm{~nm}$ on the silicon dioxide $\left(\mathrm{SiO}_{2}\right)$ surfaces, instead of shallow nano-grooves, ${ }^{15}$ could minimize undesired contribution of pentacene crystals onto the PR patterns to the charge-carrier transport, providing the perfectly confined geometries to investigate the $\pi$-conjugated nanostructure of pentacene affecting the charge transport along the source-drain $(S-D)$ electrode direction.

We then compared field-effect mobility $\left(\mu_{\mathrm{FET}}\right)$ values of OTFTs containing pentacene crystals percolated in the confined patterns with those of the standard OTFTs. As the line spacing $\left(L_{S}\right)$ between the tall PR reliefs decreased to below the average size of typical pentacene grains on smooth and organo-compatible dielectrics, $\mu_{\mathrm{FET}}$ values of

\footnotetext{
${ }^{1}$ Department of Materials Science and Engineering, Pohang University of Science and Technology (POSTECH), Pohang, Republic of Korea; ${ }^{2}$ Department of Nano, Medical and Polymer Materials, Yeungnam University, Gyeongsan, Republic of Korea and ${ }^{3}$ Department of Advanced Fiber Engineering, Inha University, Incheon, Republic of Korea

Correspondence: Professor T-W Lee, Department of Materials Science and Engineering, Pohang University of Science and Technology (POSTECH), San 31, Hyoja-dong, Nam-gu, Pohang, Gyeongbuk 790-784, Republic of Korea.

E-mail: twlee@postech.ac.kr
}

Received 5 July 2013; revised 27 November 2013; accepted 9 December 2013 
top-contacted electrode OTFTs were considerably improved. Specifically, the $\mu_{\mathrm{FET}}$ values for the $250 \mathrm{~nm}$ confined dielectric system were much higher $\left(0.33 \mathrm{~cm}^{2} \mathrm{~V}^{-1} \mathrm{~s}^{-1}\right)$ than those of the micro-scale confined or flat dielectric systems $\left(\leqslant 0.15 \mathrm{~cm}^{2} \mathrm{~V}^{-1} \mathrm{~s}^{-1}\right)$. These improvements were related to enhancement of charge-carrier transport along the $S-D$ electrodes oriented parallel to the PR lines, owing to the anisotropic intermolecular $\pi$-conjugation of pentacene nucleated from the nano-confined PR edges, providing important insight for the design of high-performance nano-scale organic electronics.

\section{EXPERIMENTAL PROCEDURE}

To demonstrate how the morphology of pentacene grown in confined regions affects charge transport, top-contact OTFTs were designed to contain nominally 50-nm-thick pentacene polycrystals confined onto poly(methyl methacrylate) (PMMA)-coated $\mathrm{SiO}_{2}$ dielectrics between periodically patterned PR reliefs. First, PR films (AZ Electronic Materials USA Co., Somerville, NJ, USA) were spin-cast on heavily doped silicon wafers (Arsenic doped n-type $\mathrm{Si}$ ) covered with thermally grown 100 -nm-thick $\mathrm{SiO}_{2}$ layers. Elastomeric PDMS masks were placed in direct contact with the PR films to form the micro- and nano-relief patterns by combining conventional photolithography with soft lithography (known as phase-shift lithography; see Supplementary Figure S1 in the Supporting Information). As the PDMS masks used in this study are transparent to ultraviolet light with a wavelength $(\lambda)$ of $>300 \mathrm{~nm}$, the transmitted light identified by the refractive index of the PDMS $(\sim 1.43)$ is modulated with an in-phase mode, within the depth of the reliefs on the bottom side of the PDMS surface. In this case, minimum doses of the transmitted light appeared at the relief edges. ${ }^{16-18}$ Values of $L_{\mathrm{S}}$, as the determining factor of the geometrical confinement, could be controlled to be $9 \mu \mathrm{m}, 3 \mu \mathrm{m}, 1 \mu \mathrm{m}$ and $250 \mathrm{~nm}$ (see Figure 1 and Supplementary Figure S2). The patterned $\mathrm{PR} / \mathrm{SiO}_{2}$ dielectrics were then treated with oxygen plasma $\left(\mathrm{O}_{2}\right.$ $50 \mathrm{sccm}, 30 \mathrm{mTorr}$ ) to eliminate the $\mathrm{PR}$ residues on the confined $\mathrm{SiO}_{2}$ regions, and ultrathin PMMA ( $\mathrm{Mw} \sim 350000 \mathrm{~g} \mathrm{~mol}^{-1}$ ) layers were spin-cast onto the dielectrics from $0.02 \mathrm{wt} \%$ toluene solution.

Pentacene (Polysis Pure Products Co., Seoul, Republic of Korea) was deposited on the substrates by an organic molecular-beam deposition system (with a quartz crystal balance to monitor nominal film thickness; under a base pressure of $\sim 10^{-6}$ Torr and substrate temperature of $30^{\circ} \mathrm{C}$ ). Top-contacted Au electrodes were fabricated on the pentacene films, specific orientations of either parallel $(P L)$ or perpendicular $(P P)$ with respect to the PR reliefs (see Figure 2). Grazing-incidence X-ray diffraction (GIXD) was performed at the 10 C1 beamline $(\lambda=1.54 \AA)$ of the Pohang Accelerator Laboratory, and atomic force microscopy (AFM) was conducted using a scanning probe microscope

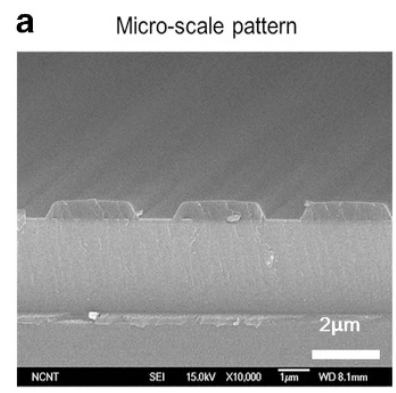

b Nano-scale pattern
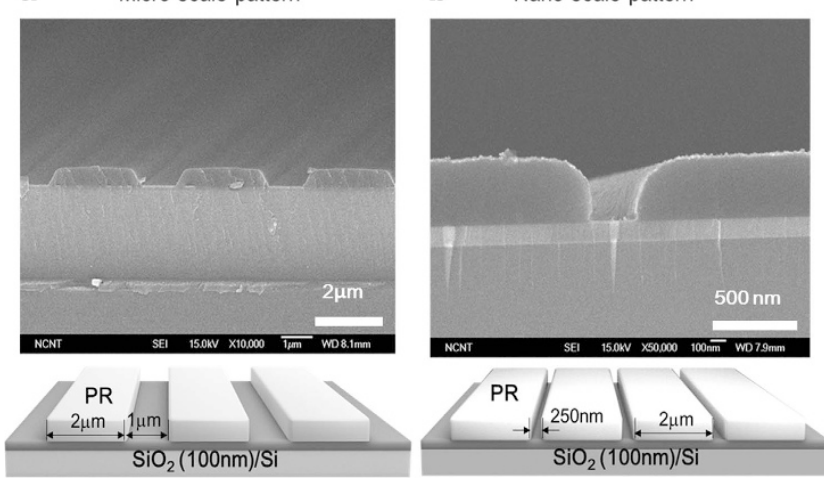

Figure 1 Scanning electron microscopy (SEM) images of photoresist (PR) pattern. (a) Micro-scale patterns consist of similarly spaced PR and silicon dioxide $\left(\mathrm{SiO}_{2}\right)$ surface-modified poly(methyl methacrylate) (PMMA) thin-film alternately. (b) Nano-scale patterns consist of $2 \mu \mathrm{m}$ width PR and $\sim 250 \mathrm{~nm}$ $\mathrm{SiO}_{2}$ layer, alternately. The heights of micro- and nano-scale patterns are $450 \mathrm{~nm}$ and $250 \mathrm{~nm}$, respectively.
(Multimode 8, Bruker, Santa Barbara, CA, USA) operated in tapping mode. The current-voltage $(I-V)$ characteristics were measured under a nitrogen conditions using a Keithley 4200 semiconductor parameter analyzer (Keithely Instruments Inc., Cleveland, OH, USA).

\section{RESULTS AND DISCUSSION}

Self-assembly of organic semiconductors to form $\pi$-conjugated orientation preferential to the charge transport direction may be one of the best methods of achieving high-performance OTFTs. ${ }^{15}$ To determine the effects of confinement on the crystal growth of pentacene showing terrace-like grains (with an average size of $1.7 \mu \mathrm{m}$ on the PMMA-coated $\mathrm{SiO}_{2}$ dielectrics; see Figure 3a) and the electrical properties of the resulting crystals in OTFT, various confined geometries on the PMMA-coated $\mathrm{SiO}_{2}$ dielectrics were manipulated by line-patterned PR reliefs $L_{\mathrm{S}}=250 \mathrm{~nm}, 1 \mu \mathrm{m}, 3 \mu \mathrm{m}$ and $9 \mu \mathrm{m}$, respectively (see Figure 1 and Supplementary Figure S2). Because the PR reliefs with a constant periodicity were much taller $(250-450 \mathrm{~nm})$ than the $\mathrm{SiO}_{2}$ dielectric layer, the gate voltage $\left(V_{\mathrm{G}}\right)$-induced electric field within PMMA-coated $\mathrm{PR} / \mathrm{SiO}_{2}$ regions was much lower than that within the PMMA-coated $\mathrm{SiO}_{2}$ dielectrics. As a result, the PR patterned geometries provided environmental conditions that enabled investigation of the charge transport behavior of OTFTs containing the pentacene crystals fabricated on the confined geometries (discussed below). However, rough PR surfaces were found to cause less-ordered pentacene aggregates with small grains $(200-300 \mathrm{~nm})$ even after the organo-compatible PMMA layers were introduced (see Figure $3 b)$.

Here, two types of top-contacted Au electrodes were fabricated to be oriented with either $P L$ or $P P$ to the charge transport direction (see Figure 2). As values of $L_{S}$ with the $\mathrm{PMMA}-\mathrm{SiO}_{2}$ surfaces decreased to below a critical value (expected to be $\sim 1.7 \mu \mathrm{m}$ ), the growth mode of pentacene was found to be a three-dimensional (3D) island instead of Stranski-Krastanov mode (or layer-by-island). Interestingly, the highest value of $\mu_{\mathrm{FET}}$ was indicated for OTFTs containing pentacene crystals percolated in nano-confined geometry (with $L_{S}=250 \mathrm{~nm}$ ) in comparison with other OTFTs. Below, we discuss how the confinement-driven crystal growth of pentacene affects the charge transport behavior along the $S-D$ electrodes.

Micro-scale $\mathrm{PR} / \mathrm{SiO}_{2}$ substrates fabricated using conventional photo-lithography contained periodically alternated $\mathrm{PR}$ and $\mathrm{SiO}_{2}$ regions (with values of $L_{\mathrm{S}}$ ranging from 1 to $9 \mu \mathrm{m}$; see Supplementary Figure S2). In contrast, periodic PR line patterns with a $2-\mu \mathrm{m}$ pitch and $L_{S}=250 \mathrm{~nm}$ (see Figure 1b) could be manipulated on the $\mathrm{SiO}_{2}$ dielectrics using phase-shift lithography as described in the Supporting Information (see Supplementary Figure S1). Because all PR line patterns possessed considerable heights, these confined patterns were expected to have significant effects on the $\pi$-conjugated self-assembly of pentacene along the long axis of the PR patterns oriented either $P L$ or $P P$ to the charge transport direction, that is, the $S-D$ electrode direction. Pentacene films were developed with a layerby-island growth mode on the PMMA-coated $\mathrm{SiO}_{2}$ dielectrics, showing terrace-like crystals with an average grain size of $\sim 1.7 \mu \mathrm{m}$, whereas on the PMMA-coated PR surfaces, 3D pentacene grains (200-300 nm) were observed (see Figure 3). In addition, the layer-byisland growth mode of pentacene tended to be changed to a 3D island growth at $L_{\mathrm{S}}<$ normal grain size $(\sim 1.7 \mu \mathrm{m}$; see Supplementary Figures S3 and S4).

The electrical performance of pentacene TFTs based on the microand nano-confined geometries was compared with that of control devices on both the PMMA-coated $\mathrm{SiO}_{2}$ and PR-SiO 2 dielectrics. In addition, variations in charge transport depending on the $S-D$ 
a

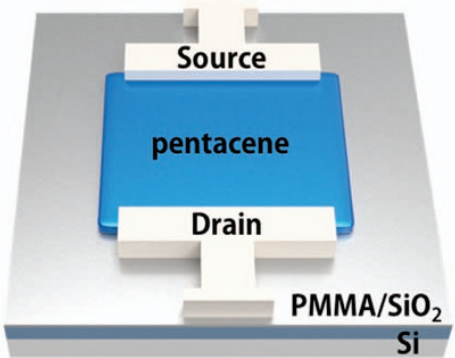

c

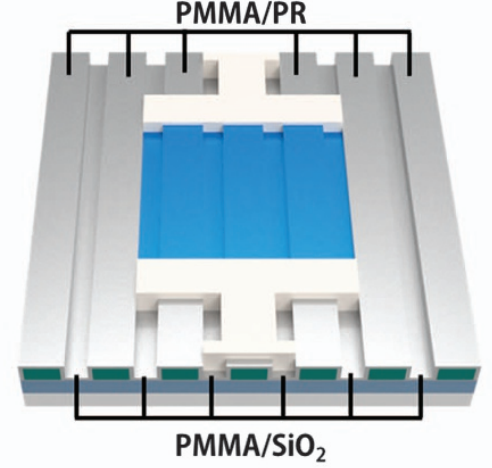

e

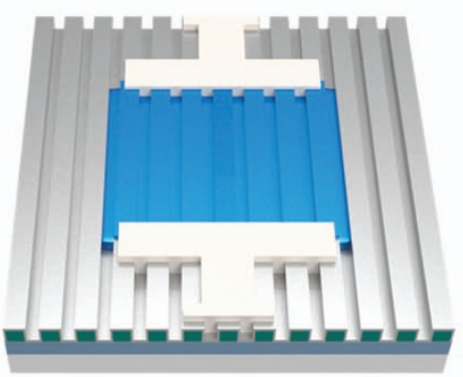

b

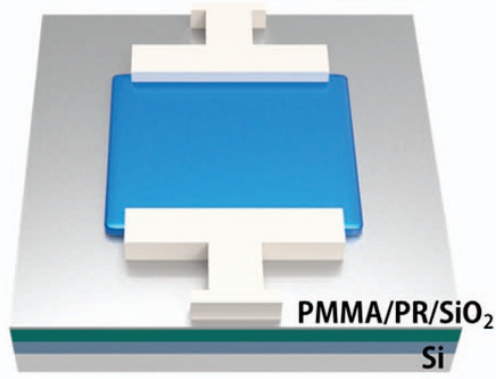

d

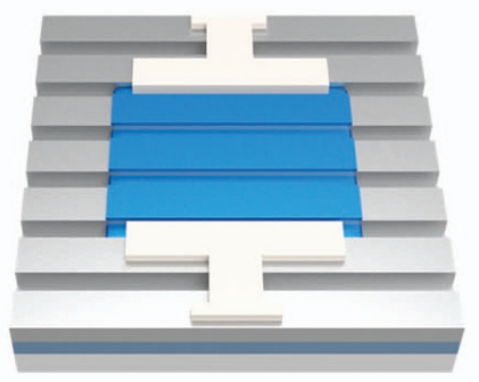

f

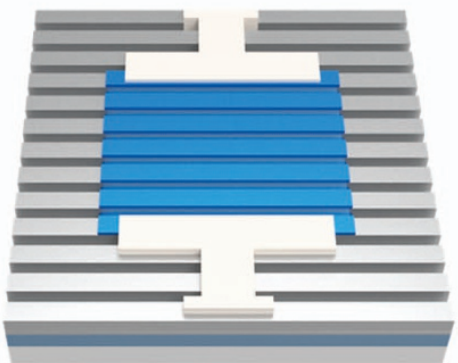

Figure 2 Schematic of the device configurations. Nonpatterned devices employed (a) the poly(methyl methacrylate) $\left(\mathrm{PMMA}^{-}\right.$-coated silicon dioxide $\left(\mathrm{SiO} \mathrm{O}_{2}\right)$ or (b) PMMA-coated photoresist (PR) film as dielectrics. Patterned devices have (c, d) micro-spaced or (e, f) nano-spaced PR line patterns. (c, e) Parallel (PL) and $(\mathbf{d}, \mathbf{f})$ perpendicular $(P P)$ stand for the directional relation between the charge transport and line pattern direction. The devices are classified according to the line pattern width and the direction between the charge transport and patterned line direction.
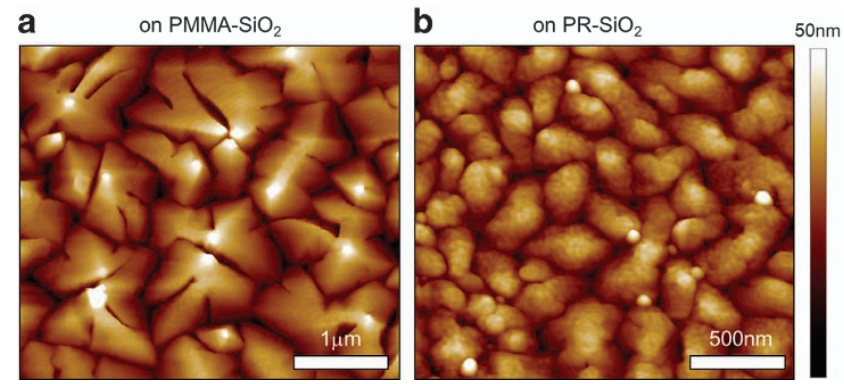

Figure 3 Atomic force microscopy (AFM) images of pentacene film (a) on the poly(methyl methacrylate) (PMMA)-coated silicon dioxide $\left(\mathrm{SiO}_{2}\right)$ surface and (b) PMMA-coated photoresist (PR) film surface. The average grain size of pentacene is $1.7 \mu \mathrm{m}$ on the $\mathrm{SiO}_{2}$ surface and $\sim 200 \mathrm{~nm}$ on the PR film.

electrode direction, that is, $P L$ and $P P$ with respect to the PR patterns, were investigated (see Figure 4). It has been reported that shallow $(\sim 30 \mathrm{~nm})$ periodic patterns induce preferred $\pi$-conjugation of pentacene along the $S-D$ electrodes (parallel to the line patterns) during film growth in OTFT; ${ }^{15}$ in this case, the charge transport was drastically enhanced and showed much higher $\mu_{\mathrm{FET}}$ values, relative to the preferred $\pi$-conjugation perpendicular to the $S-D$ electrode. The pentacene OTFTs on the PMMA-coated $\mathrm{PR} / \mathrm{SiO}_{2}$ dielectrics with a high surface roughness (root-mean-square roughness, $R_{\mathrm{q}}=5.63 \mathrm{~nm}$; channel length $=100 \mu \mathrm{m}$; channel width $=1500 \mu \mathrm{m}$ ) yielded poor $\mu_{\mathrm{FET}}$ values of $0.017 \mathrm{~cm}^{2} \mathrm{~V}^{-1} \mathrm{~s}^{-1}$, resulting from the less-ordered $\pi$-conjugation of pentacene (see Figure $3 \mathrm{~b}$ ) that was also determined by GIXD (see Figure 5).

When the drain current $\left(I_{\mathrm{D}}\right)$-gate voltage $\left(V_{\mathrm{G}}\right)$ transfer curves of the control devices based on the PMMA-coated $\mathrm{SiO}_{2}$ and $\mathrm{PR} / \mathrm{SiO}_{2}$ dielectrics were compared, the contribution of the pentacene grains percolated on the PMMA-SiO 2 sides to the total $I_{D}$ (that is, charge transport) was almost two orders of magnitude higher than that on the PMMA-PR/SiO 2 sides; for top-contact $P L$ electrode OTFTs, the charge transport from the $\mathrm{PR} / \mathrm{SiO}_{2}$ regions could be negligible. To calculate $\mu_{\mathrm{FET}}$ in the micro- and nano-scale confined systems, the active conducting channel width $(W)$, excluding the $\mathrm{PR} / \mathrm{SiO}_{2}$ regions, was used as follows: for this calculation, the values of $W$ for the PRpatterned $\mathrm{SiO}_{2}$ dielectrics were $675 \mu \mathrm{m}$ (for $L_{\mathrm{S}}=9 \mu \mathrm{m}$ ), $502.5 \mu \mathrm{m}$ (for $\left.L_{\mathrm{S}}=3 \mu \mathrm{m}\right), 375 \mu \mathrm{m}$ (for $\left.L_{\mathrm{S}}=1 \mu \mathrm{m}\right)$ and $136 \mu \mathrm{m}$ (for $L_{\mathrm{S}}=250 \mathrm{~nm}$ ), respectively.

The $I-V$ curves of the pentacene TFTs were measured in darkness at room temperature in a nitrogen atmosphere. $I_{D}-V_{G}$ transfer 
a
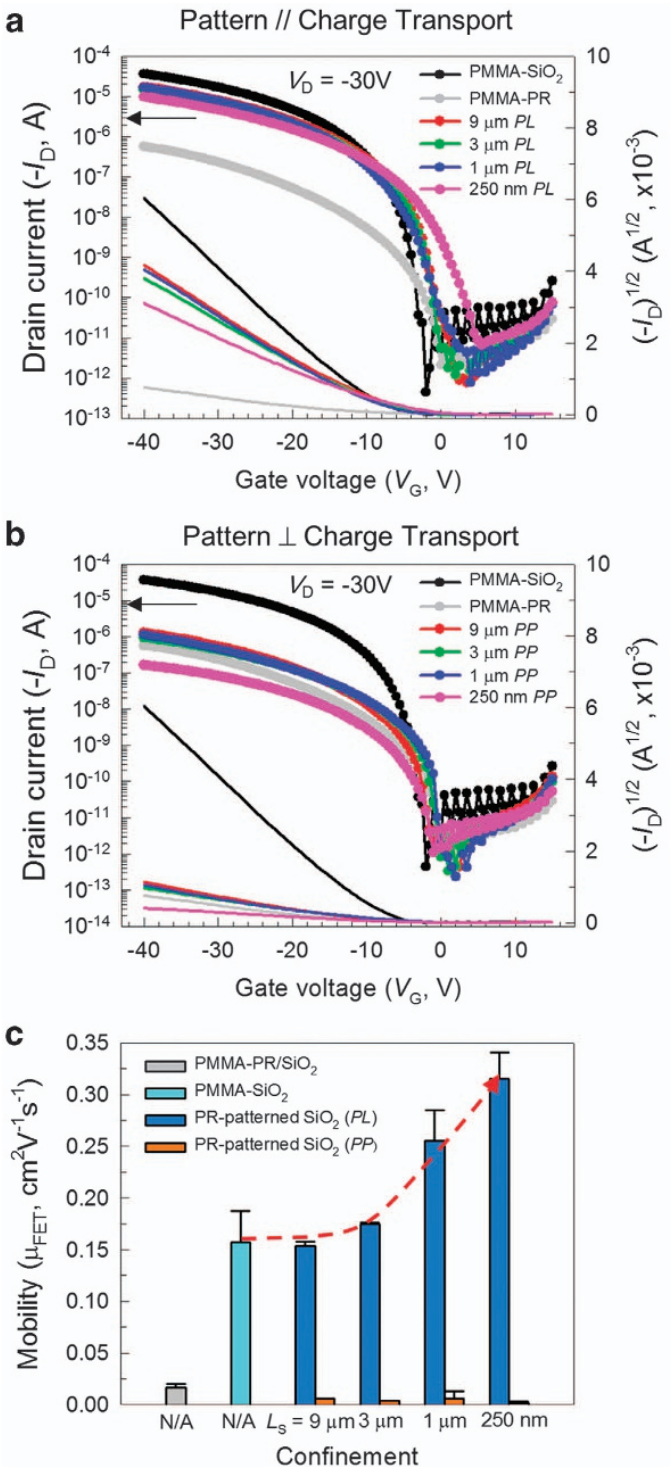

Figure 4 Electrical transfer characteristics (a, b) and field-effect mobility (c) of pentacene thin-film transistors (TFTs) within confined regions having different spaces. Devices with the direction of patterns (a) parallel and (b) perpendicular to the direction of charge transport in the channel. Gate voltage was swept at constant drain-source voltage of $-30 \mathrm{~V}$. The field-effect mobilities of 9 and $3 \mu \mathrm{m}$ patterned devices were similar to those of the nonpatterned device (poly(methyl methacrylate) (PMMA)-coated silicon dioxide $\left(\mathrm{SiO}_{2}\right)$ ) because the wider patterns have no effect of the confined region, but the field-effect mobilities of $1 \mu \mathrm{m}$ and nanometer-scale patterned devices were higher than those of nonpatterned device.

characteristics (see Figures $4 \mathrm{a}$ and $\mathrm{b}$ ) of the OTFTs were measured at a constant drain voltage $\left(V_{D}\right)$ of $-30 \mathrm{~V}$ (that is, in the saturation regime), and values of $\mu_{\mathrm{FET}}$ were calculated in this regime. ${ }^{19-21}$ Other electrical parameters such as the on/off current ratio $\left(I_{\mathrm{on}} / I_{\text {off }}\right)$, threshold voltage $\left(V_{\mathrm{T}}\right)$ and sub-threshold swing $(S S)$ were extracted from Figure 4 and summarized in Table 1 . As $L_{S}$ decreased down to $3 \mu \mathrm{m}$, values of $\mu_{\mathrm{FET}}$ for the top-contacted $P L$ electrode OTFTs seemed to be steady. However, below $L_{S}=3 \mu \mathrm{m}$, they were suddenly increased. Particularly, $\mu_{\mathrm{FET}}$ value of the nano-scale confined pentacene TFT $\left(L_{S}=250 \mathrm{~nm}\right), 0.333 \mathrm{~cm}^{2} \mathrm{~V}^{-1} \mathrm{~s}^{-1}$ was higher than that $\left(0.151 \mathrm{~cm}^{2} \mathrm{~V}^{-1} \mathrm{~s}^{-1}\right)$ of the control device on the PMMA-coated $\mathrm{SiO}_{2}$ dielectric. This result suggests that the nano-confined geometry along

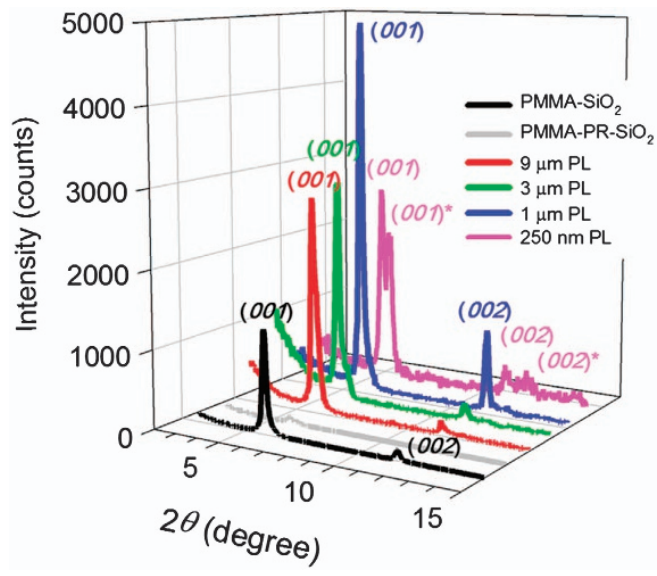

Figure 5 Grazing-incidence X-ray diffraction (GIXD) patterns for pentacene film with 50-nm thickness deposited on the poly(methyl methacrylate) (PMMA)-coated silicon dioxide $\left(\mathrm{SiO}_{2}\right)$, PMMA-coated photoresist (PR) film and PMMA-coated patterned $\mathrm{PR} / \mathrm{SiO}_{2}$ surfaces. We applied the geometric factor of the confined line spaces made by PR to calculate the data of GIXD.

the charge transport direction induces an anisotropic $\pi$-conjugated self-assembly of pentacene along the $S-D$ electrodes. As shown in Figure $4 \mathrm{c}$, the transition in $\mu_{\mathrm{FET}}$ was indicated at $L_{\mathrm{S}}=1 \mu \mathrm{m}$, and related to the crystal development of pentacene in the confined geometries; in this case, preferred crystal growing can be along the charge transport direction.

To interpret the relationship between the confinement-driven crystal structure and charge transport, AFM and GIXD analyses were systematically performed for various thick pentacene films on all of the dielectric surfaces. AFM topographies first indicated that pentacene films on PMMA-coated $\mathrm{SiO}_{2}$ dielectrics grew in a manner that differed from that on $\mathrm{PR} / \mathrm{SiO}_{2}$ dielectrics (see Supplementary Figure S5). As shown in Supplementary Figure S5a, typical layerby-island-grown pentacene crystals were observed on the PMMAcoated $\mathrm{SiO}_{2}$ surfaces. ${ }^{22}$ In this case, pentacene molecules were more strongly bound to the substrate than to each other $\left(W_{\mathrm{A}}>2 \gamma_{\mathrm{p}}\right.$, where $W_{\mathrm{A}}$ is the work of adhesion and $\gamma_{\mathrm{p}}$ is the surface energy of the pentacene crystal plane). and the seeding molecules are condensed to form the first molecular layer $(M L)$ on the surface. ${ }^{23}$ For layer growth (or island growth), $\gamma_{i}+\gamma_{p}<\gamma_{g}\left(\right.$ or $\gamma_{i}+\gamma_{p}>\gamma_{g}$ ). Here, $\gamma_{i}, \gamma_{p}$ and $\gamma_{g}$ are the interfacial energy, surface energy of pentacene thin-film and gate dielectrics, respectively. If we introduce the term, work of adhesion $W_{\mathrm{A}}$, then $W_{\mathrm{A}}=\gamma_{\mathrm{p}}+\gamma_{\mathrm{g}}-\gamma_{\mathrm{i}}$. By eliminating $\gamma_{\mathrm{i}}, W_{\mathrm{A}}>2 \gamma_{\mathrm{p}}$ $\left(W_{\mathrm{A}}<2 \gamma_{\mathrm{p}}\right)$ for layer-by-layer growth (or island growth). The $2 \gamma_{p}$ is work of cohesion of pentacene. In contrast, on the rough PMMA-coated $\mathrm{PR} / \mathrm{SiO}_{2}$ dielectrics, 3D aggregates started to be grown at an initial stage without forming $M L$, that is, with a 3D island growth mode (see Supplementary Figure S5b), but they showed irregular grains with a nearly amorphous structure (determined by GIXD, see Figure 5), explaining the poor electrical performance $\left(0.017 \mathrm{~cm}^{2} \mathrm{~V}^{-1} \mathrm{~s}^{-1}\right)$. The surface heterogeneity of gate dielectrics also affects the crystal growth of organic semiconductors. ${ }^{24-30}$ On the 250-nm and $1 \mu \mathrm{m}$-confined PR-patterned $\mathrm{SiO}_{2}$ dielectrics, pentacene started to be nucleated at the bottom-contacted edges of the PR reliefs as thermodynamically active nucleation sites. ${ }^{15,31}$ The pentacene grains were also reported to be elongated along the longer axis (less-confined direction) of the nano-confined geometry. ${ }^{15}$ The anisotropic grain morphology of pentacene could be observed for several nm-thick pentacene films on the 250-nm-confined system (see Supplementary Figures S3 and S4). 
Table 1 Extracted other electrical parameters of pentacene FETs

\begin{tabular}{|c|c|c|c|c|c|c|c|c|c|c|}
\hline Parameter & $\mathrm{SiO}_{2} \mathrm{film}$ & PR film & $9 \mu m P L$ & $9 \mu m P P$ & $3 \mu \mathrm{mPL}$ & $3 \mu m P P$ & $1 \mu \mathrm{m} P L$ & $1 \mu m P P$ & $n m P L$ & $n m P P$ \\
\hline SS & 0.667 & 3.468 & 1.174 & 1.908 & 0.978 & 1.854 & 1.454 & 1.902 & 1.987 & 3.717 \\
\hline Mobility & 0.151 & 0.020 & 0.158 & 0.006 & 0.176 & 0.004 & 0.279 & 0.006 & 0.333 & 0.001 \\
\hline
\end{tabular}

Abbreviations: FETs, field-effect transistors; $I_{\text {on }} / I_{\text {off }}$, on/off current ratio; Mobility, field-effect mobility $\left(\mathrm{cm}^{2} \mathrm{~V}^{-1} \mathrm{~s}^{-1}\right)$; PL, parallel; PP, perpendicular; PR, photoresist; SiO 2 , silicon dioxide; $S S$, subthreshold swing $\left(\mathrm{V} \mathrm{dec}^{-1}\right) ; V_{\mathrm{T}}$, threshold voltage $(\mathrm{V})$.
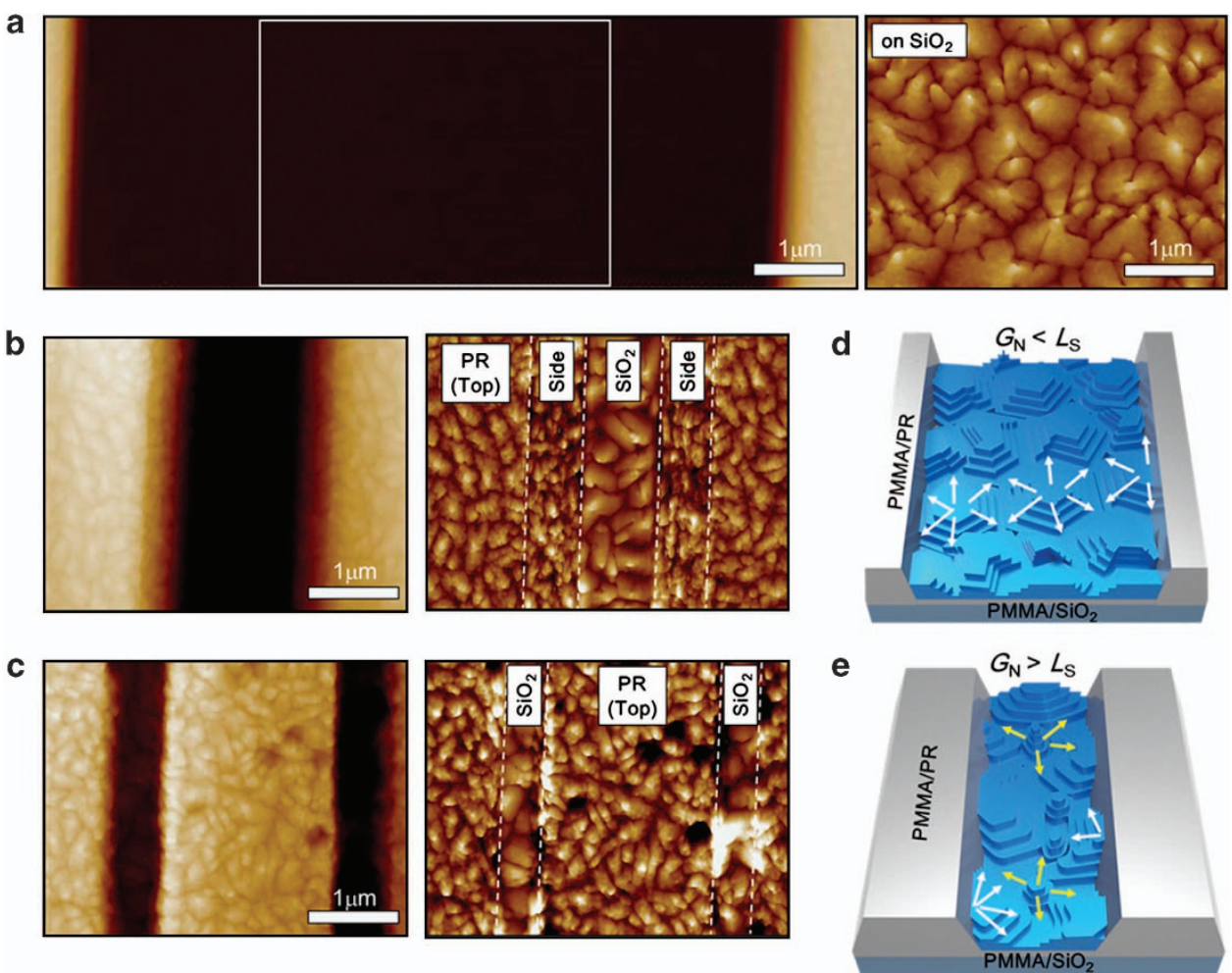

Figure 6 (a-c) Atomic force microscopy (AFM) topographies of 30-nm-thick pentacene thin films on the different photoresist (PR) patterned substrates: (a) $L_{S}=9 \mu \mathrm{m}$; (b) $L_{S}=1 \mu \mathrm{m}$; (c) $L_{S}=250 \mathrm{~nm}$, respectively. (d, e) Schematic illustration of pentacene growth in the confined region.

As mentioned in the Experimental Section, the successive procedures of PR patterning, $\mathrm{O}_{2}$-plasma treatment and PMMA coating could form the patterned PR barriers with a height (micro-pattern: $\sim 450 \mathrm{~nm}$; nano-pattern: $\sim 250 \mathrm{~nm}$ ) sufficient to produce the perfectly confined $\mathrm{SiO}_{2}$ dielectric surfaces with smooth and organocompatible characteristics. It should be noted that some pentacene morphologies were not clearly detected using AFM with a polygonalshaped probe tip because of the deep valley-like geometries; therefore, scanning electron microscopy was also used as a complementary tool (see Supplementary Figure S4). AFM analyses of the pentacene films on the PMMA-coated PR-patterned $\mathrm{SiO}_{2}$ dielectrics revealed that the crystal growth mechanism of pentacene could be changed via controlling the degree of the geometrical confinement. When $L_{\mathrm{S}}$ is sufficiently wider than the normal grain size $\left(G_{\mathrm{N}}\right)$ of pentacene, crystal nucleation of pentacene occurs simultaneously at both the PR barrier edges and PMMA-coated $\mathrm{SiO}_{2}$ surfaces (see Supplementary Figure S6) and then the crystals are growing laterally with a radial direction until they are percolated with the neighbors (see arrowmarked directions in Figures $6 \mathrm{~d}$ and e). After the lateral crystal percolation, vertical crystal growth becomes dominant, showing pentacene crystals with vertically terrace-like steps (see Figures 6a and d). In contrast, when $L_{S}<G_{\mathrm{N}}$, most nuclei seem to appear at the PR barrier edges and the crystal growing prefers the less-confined open space perpendicular to the PR barriers, indicating elongated crystal grains along the growing direction (see Supplementary Figures S3 and S4). In OTFTs, charge-carrier transport is limited mainly within a several-nm-thick semiconducting layer that directly contacts the dielectric. ${ }^{32-35}$ As the pentacene film becomes thicker, vertically grown crystalline islands are percolated in comparison with the irregular aggregates on the top-most PR surfaces (see Figures 6b, c and e). As a result, suggesting that the unconventional crystal growth induces anisotropic $\pi$-overlap of pentacene along the percolated crystals in the nano-confined geometries.

In addition, 1D out-of-plane GIXD profiles were obtained for the 50-nm-thick pentacene films deposited on all of the dielectrics (see Figure 5). All out-of-plane X-ray peaks were indexed as (O0l) and $(00 l)^{*}$ crystal planes originating from the 'thin film phase' with a pseudo-orthorhombic unit cell and the 'bulk phase' with a triclinic unit cell, respectively. The inter-planar spacing of (001) was $d_{(001)}=15.3 \AA$ and $\mathrm{d}_{(001)^{*}}=$ and $14.2 \AA .^{36,37}$ It should be noted that 
the lateral width of the incident X-ray beam used for GIXD $(<300 \mu \mathrm{m})$ was much wider than all of the $L_{S}$, and the resulting GIXD profiles contained the crystal information on both the PMMAcoated $\mathrm{PR} / \mathrm{SiO}_{2}$ and $\mathrm{SiO}_{2}$ regions. Fortunately, it was found that the pentacene aggregates on the topmost $\mathrm{PR} / \mathrm{SiO}_{2}$ surfaces had almost amorphous-like structure, as determined by GIXD analysis (gray solid lines in Figure 5).

Interestingly, the peak intensities of X-ray reflections from the micro- and nano-scale confined systems were much higher than those from the PMMA-coated $\mathrm{SiO}_{2}$ system. Particularly, the 1D GIXD profile of the 250-nm-confined sample showed the X-ray reflections of both $(00 l)$ and $(O 0 l)^{*}$ crystal planes with abnormally high intensities owing to its lower volume fraction of the nano-confined regions of $\sim 0.09(=136 \mu \mathrm{m} / 1500 \mu \mathrm{m})$. The confinement effect decreased with an increase in $L_{S}$. There are two main factors affecting the X-ray reflections: scattering volume and crystallinity. The crystal growth of pentacene on the smooth $\mathrm{PMMA}-\mathrm{SiO}_{2}$ surface might be much faster than that on the uneven PMMA-PR surface. In this case, the actual thicknesses of the pentacene crystals grown in the confined area might be thicker than the nominal thicknesses monitored by the Quartz crystal balance during film deposition. However, the highly ordered X-ray reflections of the $1-\mu \mathrm{m}$ patterned sample could not be explained only with the scattering volume effect, suggesting the following possibility; the asymmetrically micro- and nano-confined geometries can drive the self-assembly of pentacene molecules to form highly $\pi$-conjugated and crystalline structures, in comparison with flat or less-confined geometries, where there exist random nucleation sites and radial crystal growth of organic semiconductors.

For example, the dendritic- or pyramid-shaped crystals of pentacene on the PMMA-coated $\mathrm{SiO}_{2}$ surface had many lateral grain boundaries (see Figure $6 \mathrm{a}$ ), where the $\pi$-conjugation between the molecules is intrinsically mismatched. In contrast, pentacene started to grow initially at the PR edges, forming percolated crystal layer (related to $(00 l)$ crystal reflection), and further grew into highly compact crystals (see yellow-marked directions in Figure 6e): in this case, the portion of the 'bulk phase' corresponding to $(00 l)^{*}$ reflections tends to increase during filling the confined geometry (see Figures $6 \mathrm{~b}$ and c).

Therefore, the morphological and X-ray results strongly support that inside the asymmetrically confined geometries, pentacene is crystallized from the patterned PR edges and its crystals contain $\pi$-conjugated planes preferentially oriented to the charge transport direction; the $\pi-\pi$ stacking of pentacene molecules in the direction of charge transport increased as the patterned spaces became narrower. This type of confinement-induced molecular stacking of pentacene enhanced the charge transport in OTFT. As a result, the field-effect mobility of the device made of pentacene films grown in the $250-\mathrm{nm}$ patterned region was approximately two times greater than those of the devices made from films grown in the unpatterned and micro (9-3 $\mu \mathrm{m})$-patterned region.

\section{CONCLUDING REMARKS}

We analyzed how the morphologies of organic semiconductor films confined in the micro- and nano-scale line and space relief patterns affect charge transport. As the patterned space region for confining the growth of organic semiconductors decreased below the average grain size $(\sim 1.7 \mu \mathrm{m})$, the field-effect mobility increased by improving molecular ordering in the direction of charge transport because of increased confinement in the relief pattern. The field-effect mobility of the pentacene TFT in nano-confined regions $(250 \mathrm{~nm})$ was higher $\left(0.333 \mathrm{~cm}^{2} \mathrm{~V}^{-1} \mathrm{~s}^{-1}\right)$ than that of the normal thin-film device $\left(0.151 \mathrm{~cm}^{2} \mathrm{~V}^{-1} \mathrm{~s}^{-1}\right)$. We observed a transition of the field-effect mobility at $\sim 1 \mu \mathrm{m}$ for the confined pattern, in which the patterned space is smaller than the normal grain size in thin films $(\sim 1.7 \mu \mathrm{m})$. This transition indicates that molecular ordering is influenced by the pattern's confining effect on pentacene film growth. The results presented herein provide an approach that can be used to further increase the charge transport mobility of polycrystalline organic semiconductors by increasing the molecular ordering in the direction of charge transport. Furthermore, the organic semiconductor well confined in nano-structures is very similar to $1 \mathrm{D}$ nanowires because tall PR patterns prohibit charge transport perpendicular to the direction of $S-D$ electrodes. The increased mobility of the transistors using pentacene grown in $1 \mathrm{D}$ nano-confined regions implies that 1D nanostructures of organic semiconductors can possess better molecular ordering and thus higher mobility than devices containing their thin-film counterparts.

\section{ACKNOWLEDGEMENTS}

This work was supported a grant (Code No. 2013M3A6A5073175) from the Center for Advanced Soft Electronics under the Global Frontier Research Program of the Ministry of Science, ICT and Future Planning, Korea. This research was also supported by the Pioneer Research Center Program through the National Research Foundation (NRF) of Korea funded by the Ministry of Science, ICT and Future Planning (2012-0009460) and supported by Basic Science Research Program through the National Research Foundation of Korea funded by the Ministry of Education (NRF-2013R1A1A2012660).

1 Knipp, D., Street, R. A., Volkel, A. \& Ho, J. Pentacene thin film transistors on inorganic dielectrics: morphology, structural properties, and electronic transport. J. Appl. Phys. 93, 347-355 (2003).

2 Lee, H. S., Kim, D. H., Cho, J. H., Park, Y. D., Kim, J. S. \& Cho, K. Enhancement of interconnectivity in the channels of pentacene thin-film transistors and its effect on field-effect mobility. Adv. Funct. Mater. 16, 1859-1864 (2006).

3 Ruiz, R., Nickel, B., Koch, N., Feldman, L. C., Haglund, R. F., Kahn, A., Family, F. \& Scoles, G. Dynamic scaling, island size distribution, and morphology in the aggregation regime of submonolayer pentacene films. Phys. Rev. Lett. 91, 136102 (2003).

4 Shin, K., Yang, C., Yang, S. Y., Jeon, H. \& Park, C. E. Effects of polymer gate dielectrics roughness on pentacene field-effect transistors. Appl. Phys. Lett. 88, 072109 (2006)

5 Shtein, M., Mapel, J., Benziger, J. B. \& Forrest, S. R. Effects of film morphology and gate dielectric surface preparation on the electrical characteristics of organic-vaporphase-deposited pentacene thin-film transistors. Appl. Phys. Lett. 81, 268-270 (2002)

6 Virkar, A., Mannsfeld, S., Oh, J. H., Toney, M. F., Tan, Y. H., Liu, G.-Y., Scott, J. C., Miller, R. \& Bao, Z. The role of OTS density on pentacene and C60 nucleation, thin film growth, and transistor performance. Adv. Funct. Mater. 19, 1962-1970 (2009).

7 Min, S.-Y., Kim, T.-S., Kim, B. J., Cho, H., Noh, Y.-Y., Yang, H., Cho, J. H. \& Lee, T.-W. Large-scale organic nanowire lithography and electronics. Nat. Commun. 4, 1773 (2013)

8 Noh, Y.-Y., Zhao, N., Caironi, M. \& Sirringhaus, H. Downscaling of self-aligned, all-printed polymer thin-film transistors. Nat. Nanotech. 2, 784-789 (2007).

9 Jiang, L., Wang, X. \& Chi, L. Nanoscaled surface patterning of conducting polymers. Small 10, 1309-1321 (2011).

10 Briseno, A. L., Mannsfeld, S. C. B., Jenekhe, S. A., Bao, Z. \& Xia, Y. Introducing organic nanowire transistors. Mater. Today 11, 38-47 (2008).

11 Oh, J. H., Lee, H. W., Mannsfeld, S., Stoltenberg, R. M., Jung, E., Jin, Y. W., Kim, J. M., Yoo, J.-B. \& Bao, Z. Solution-processed, high-performance n-channe organic microwire transistors. Proc. Natl. Acad. Sci. USA 106, 6065-6070 (2009).

12 Zaumseil, J. \& Sirringhaus, H. Electron and ambipolar transport in organic field-effect transistors. Chem. Rev. 107, 1296-1323 (2007).

13 Fabiano, S., Musumeci, C., Chen, Z., Scandurra, A., Wang, H., Loo, Y.-L., Facchetti, A. \& Pignataro, B. From monolayer to multilayer n-channel polymeric field-effect transistors with precise conformational order. Adv. Mater. 24, 951-956 (2012).

14 Schenning, A. P. H. J. \& Meijer, E. W. Supramolecular electronics; nanowires from selfassembled ?-conjugated systems. Chem. Commun. 26, 3245-3258 (2005)

15 Jo, S. J., Kim, C. S., Lee, M. J., Kim, J. B., Ryu, S. Y., Noh, J. H., Ihm, K., Baik, H. K. \& Kim, Y. S. Inducement of azimuthal molecular orientation of pentacene by imprinted periodic groove patterns for organic thin-film transistors. Adv. Mater. 20, 1146-1153 (2008) 
16 Rogers, J. A., Paul, K. E., Jackman, R. J. \& Whitesides, G. M. Using an elastomeric phase mask for sub-100?nm photolithography in the optical near field. Appl. Phys. Lett. 70, 2658-2660 (1997).

17 Maria, J., Jeon, S. \& Rogers, J. A. Nanopatterning with conformable phase masks. J. Photochem. Photobiol. A Chem. 166, 149-154 (2004).

18 Lee, T.-W., Jeon, S., Maria, J., Zaumseil, J., Hsu, J. W. P. \& Rogers, J. A. Soft-contact optical lithography using transparent elastomeric stamps: application to nanopatterned organic light-emitting devices. Adv. Funct. Mater. 15, 1435-1439 (2005).

19 Horowitz, G., Lang, P., Mottaghi, M. \& Aubin, H. Extracting parameters from the current voltage characteristics of organic field effect transistors. Adv. Funct. Mater. 14, 1069-1074 (2004).

20 Horowitz, G. Organic thin film transistors: from theory to real devices. J. Mater. Res. 19, 1946-1962 (2004)

21 Chabinyc, M. L. \& Salleo, A. Materials requirements and fabrication of active matrix arrays of organic thin-film transistors for displays. Chem. Mater. 16, 4509-4521 (2004).

22 Smith, D. L. Thin-Film Deposition: Principles and Practice Ch. 5. Deposition 119-220 (McGraw-Hill, New York, 1995).

23 Venables, J. A., Spiller, G. D. T. \& Hanbucken, M. Nucleation and growth of thin films. Rep. Prog. Phys. 47, 399-459 (1984).

24 Fritz, S. E., Kelley, T. W. \& Frisbie, C. D. Effect of dielectric roughness on performance of pentacene TFTs and restoration of performance with a polymeric smoothing layer. J. Phys. Chem. B 109, 10574-10577 (2005).

25 Weng, S.-Z., Hu, W.-S., Kuo, C.-H., Tao, Y.-T., Fan, L.-Z. \& Yang, Y.-W. Anisotropic field-effect mobility of pentacene thin-film transistor: effect of rubbed self-assembled monolayer. Appl. Phys. Lett. 89, 172103 (2006).

26 Kang, S.-J., Noh, Y.-Y., Baeg, K.-J., Ghim, J., Park, J.-H., Kim, D.-Y., Kim, J. S., Park, J. H. \& Cho, K. Effect of rubbed polyimide layer on the field-effect mobility in pentacene thin-film transistors. Appl. Phys. Lett. 92, 052107 (2008)

27 Wang, W. C., Zhong, D. Y., Zhu, J., Kalischewski, F., Dou, R. F., Wedeking, K., Wang, Y. $\&$ Heuer, A. Patterned nucleation control in vacuum deposition of organic molecules. Phys. Rev. Lett. 98, 225504 (2007).

28 Shang, L., Liu, M., Tu, D., Zhen, L., Liu, G., Jia, R., Li, L. \& Hu, W. Controllable and reproducible fabrication of high anisotropic organic field effect transistors. Thin Solid Films 516, 5093-5097 (2008).
29 Chang, M.-H., Chou, W.-Y., Lee, W.-C., Cheng, H.-L., Chung, H.-Y., Chang, C.-C., Chiu, C.-Y. \& Ho, T.-Y. Polymorphic transformation induced by nanoimprinted technology in pentacene-film early-stage growth. Appl. Phys. Lett. 97, 183301 (2010).

30 Chou, W.-Y. \& Cheng, H.-L. An orientation controlled pentacene film aligned by photoaligned polyimide for organic thin film transistor applications. Adv. Funct. Mater. 14, 811-815 (2004)

31 Anton, R., Poppa, H. \& Flanders, D. C. The effect of grooves in amorphous substrates on the orientation of metal deposits. J. Cryst. Growth 56, 433-448 (1982).

32 Fritz, S. E., Martin, S. M., Frisbie, C. D., Ward, M. D. \& Toney, M. F. Structural characterization of a pentacene monolayer on an amorphous $\mathrm{SiO} 2$ substrate with grazing incidence X-ray diffraction. J. Am. Chem. Soc. 126, 4084-4085 (2004).

33 Yoshida, H. \& Sato, N. Grazing-incidence X-ray diffraction study of pentacene thin films with the bulk phase structure. Appl. Phys. Lett. 89, 101919 (2006).

34 Jiang, Y., Qi, Q., Wang, R., Zhang, J., Xue, Q., Wang, C., Jiang, C. \& Qiu, X. Direct observation and measurement of mobile charge carriers in a monolayer organic semiconductor on a dielectric substrate. ACS Nano 5, 6195-6201 (2011).

35 Yang, H., Shin, T. J., Ling, M.-M., Cho, K., Ryu, C. Y. \& Bao, Z. Conducting AFM and 2D GIXD studies on pentacene thin films. J. Am. Chem. Soc. 127, 11542-11543 (2005).

36 Yoneya, N., Noda, M., Hirai, N., Nomoto, K., Wada, M. \& Kasahara, J. Reduction of contact resistance in pentacene thin-film transistors by carrier injection into a-few-molecular-layer channel. Appl. Phys. Lett. 85, 4663-4665 (2004).

37 Granstrom, E. L. \& Frisbie, C. D. Field effect conductance measurements on thin crystals of sexithiophene. J. Phys. Chem. B 103, 8842-8849 (1999).

\section{(c) (i) (2)(2) This work is licensed under a Creative Commons Attribution-NonCommercial-ShareAlike 3.0 Unported License. To view a copy of this license, visit http://creativecommons. org/licenses/by-nc-sa/3.0/}

Supplementary Information accompanies the paper on the NPG Asia Materials website (http://www.nature.com/am) 